\title{
FE Analyses of Eddy Currents in W7-X Plasma Vessel
}

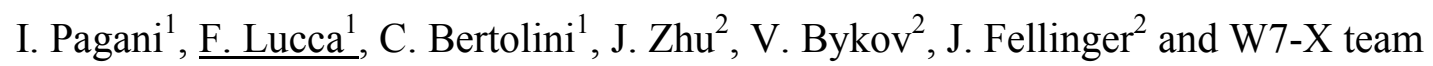 \\ ${ }^{1}$ LT Calcoli Srl, via Bergamo 60, 23807 Merate (LC), Italy \\ ${ }^{2}$ IPP- Teilinstitut Greisfald, MPI fur Plasmaphysik, Wendelsteinstraße 1, D-17491 Greifswald
}

\begin{abstract}
Wendelstein 7-X is the largest optimized stellarator, which is presently in operation in Greifswald, Germany. During possible fast plasma disruptions, relatively small plasma bootstrap current rapidly vanishes and consequently eddy currents are induced in all electrically conducting components including sophisticated Plasma Vessel (PV).

This paper focuses on the evaluation of eddy currents induced in PV components due to plasma bootstrap current decay. This task was accomplished by means of a 3D PV FE model. Due to geometry complexity, special emphasis was on specific modelling issues (elements type, mesh density and excitation representation). At first, the PV structure was modelled without 254 ports, which were included subsequently. A procedure to allow quick and easy introduction or exclusion of ports from EM analyses was devised and implemented. In this way, the influence of these structures on the eddy currents could be evaluated efficiently.

Peak value of total eddy current running around the PV structure without ports turns out to be around $20 \mathrm{kA}$ Furthermore, results indicate that the ports presence leads to a relatively minor decrease of this value, around $5 \%$. Current concentration around specific ports is highlighted.

The paper presents also two examples of re-using FEM and results.
\end{abstract}

Keywords: W7-X, Plasma Vessel, electromagnetic analyses, eddy current, plasma current decay

\section{Introduction}

Wendelstein 7-X (W7-X) is a fivefold optimized stellarator in operation in Greifswald, Germany. W7-X Plasma Vessel (PV) consists of five modules made of single shell $17 \mathrm{~mm}$ thick stainless steel and having 254 openings for ports with different thickness and length. Both the ports number and their configuration are different in each PV module.

During possible fast plasma disruptions, the plasma bootstrap current rapidly vanishes and consequently eddy currents are generated in all electrically conducting components in plasma vicinity. The evaluation and analysis of these eddy currents are of major interest, because of the electromagnetic loads resulting from interaction of the eddy current with the magnetic field created by the magnets system and by the plasma.

This paper focuses on the evaluation of the eddy currents induced in the W7-X PV components due to the decay of $100 \mathrm{kA}$ bootstrap current in the plasma (exponential decay with a time constant of 140ms) [1]. This task was accomplished by means of a 3D FE model of the PV, the excitations and the empty space around them.

The first step is devoted to the evaluation of the eddy currents in the PV without considering the effect of the ports (PV body model). Within this phase, also the impact of the mesh size, of the mesh topology and of the excitations modelling procedure is analyzed and it turned out in all cases that the influence of mentioned parameters can be considered as acceptably small.

The development of a mesh of the PV, which is already 'adapted' to the inclusion of all ports, was the next step of the activity. The results obtained on the modified model were carefully compared with the previous PV body model.

In the final phase, all the ports were introduced in the FE model and their overall effect on the eddy currents was then evaluated. Additionally, a procedure to allow quick and easy introduction or exclusion of ports from EM analyses was devised and implemented. Later, to safe computational resources during possible re-use of the model for $\mathrm{W} 7-\mathrm{X}$ component analysis, the procedure to select one ( 72 degree) or several modules with proper boundary conditions was developed and verified by comparison with the results of full 360 degree FE model.

The aim of the activity described here is to perform and benchmark a PV FE model that allows studying the behavior of diagnostic systems and other components placed inside PV shell under transient phenomena that take places during bootstrap current decay.

One typical example of the model re-use is the calculation to estimate the part of PV eddy current entering the attached cryo-pump and to analyze the resultant Lorenz forces in the component [4]. Another example is an estimation of possible influence of toroidal current fast decay as it was measured in August 2018.

\section{Analysis of eddy currents in plasma vessel without ports}

The main structure of the W7-X PV has a complex toruslike shape consisting of five identical modules each covering an angle of 72 degrees.

The solid FE model of the PV, shown with transparent elements in Fig. 1, was developed starting from the PV one sector geometry; the equivalent diameter of $\mathrm{PV}$ toroidal section is $1.62 \mathrm{~m}$ The average elements size is 
around $10 \mathrm{~cm}$; denser regions are those with very odd and irregular curvature, while for small curvature regions the mesh is coarser.

As far as the geometry of the plasma line current is concerned, this is defined parametrically. The volume in the FE model where the excitation current is applied (solid region in Fig. 1), was created by reproducing the 3D plasma current center line and then a small circle with radius of $5 \mathrm{~cm}$ was extruded along the line.

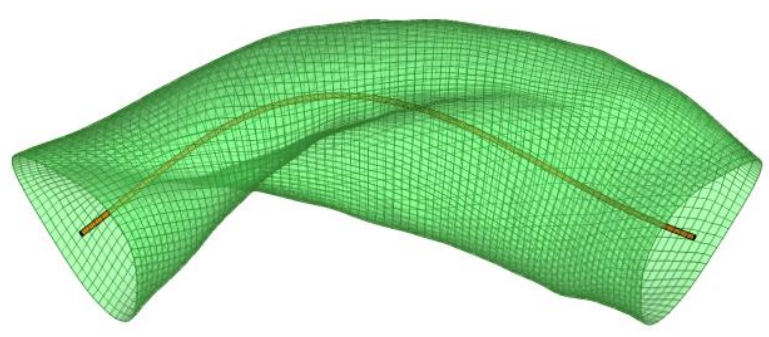

Fig. 1: 'Baseline' model of PV (transparent elements) and excitation region (solid elements)

In the FE model, also the empty space between excitations and the PV and the exterior space up to a distance of $30 \mathrm{~m}$ from the PV were modelled to simulate the electromagnetic environment correctly. At the exterior face of the model, 'open field' boundary conditions were set. At boundary planes in the toroidal direction, there are cyclical boundary conditions to reproduce the PV and excitations periodicity. All the analyses were performed in ANSYS, using SOLID97 element type [2].

Due to the complexity of the geometry of the structure to be analyzed, the special emphasis was put on modelling issues such as elements type, mesh density and excitation representation. The model described above was used as a 'baseline' model. Features and parameters of FE models employed in subsequent sensitivity analyses are summarized in Table 1. Elements number refers to whole models (conducting and non-conducting structures).

Table 1. Characteristics of FE models used for parametric analysis

\begin{tabular}{|c|c|c|c|}
\hline model & $\begin{array}{l}\text { Element } \\
\text { size }\end{array}$ & $\begin{array}{c}\text { Element } \\
\text { type/number }\end{array}$ & $\begin{array}{l}\text { Exc } \\
\text { radius }\end{array}$ \\
\hline baseline & $10 \mathrm{~cm}$ & hexa/198630 & $5 \mathrm{~cm}$ \\
\hline Finer model & $5 \mathrm{~cm}$ & hexa/432150 & $5 \mathrm{~cm}$ \\
\hline $\begin{array}{l}\text { Penta \& } \\
\text { tetra model }\end{array}$ & $10 \mathrm{~cm}$ & $\begin{array}{l}\text { PV: penta } \\
\text { Other: tetra/ } \\
1129455\end{array}$ & $5 \mathrm{~cm}$ \\
\hline $\begin{array}{l}\text { Exc bigger } \\
\text { model }\end{array}$ & $10 \mathrm{~cm}$ & hexa/198630 & $10 \mathrm{~cm}$ \\
\hline $360^{\circ}$ model & $10 \mathrm{~cm}$ & hexa/993150 & $5 \mathrm{~cm}$ \\
\hline
\end{tabular}

Fig. 2 shows the total eddy current induced in the PV (i.e. the total current running in a PV section) for all models analyzed. Compared to the baseline model, the maximum difference in total eddy current is $0.13 \%$ for finer model, while for the other models it is less than $0.1 \%$.
In addition, during W7-X operation phase (OP) called 'OP1.2b' in August 2018, the toroidal plasma current decay was observed with initial current around $20 \mathrm{kA}$ and time constant of about $2.8 \mathrm{~ms}$ (maximum current change rate $\sim 7 \mathrm{MA} / \mathrm{s}$ ) triggered by the electron cyclotron current drive (ECCD) [2]. In order to estimate the impacts on critical in-vessel components, the baseline model where re-used and the induced eddy current in the PV was calculated and compared with the result of the originally assumed plasma current decay scenario.

It turned out that the maximum total current in the PV is $16.1 \mathrm{kA}$, which is about $15.6 \%$ lower than during assumed originally most critical scenario (see Table 2). The reduction of induced current in OP $1.2 \mathrm{~b}$ is due to the reduction of plasma current (1/5 respect the reference scenario) even if is faster $(2.8 \mathrm{~ms}$ vs $140 \mathrm{~ms})$.

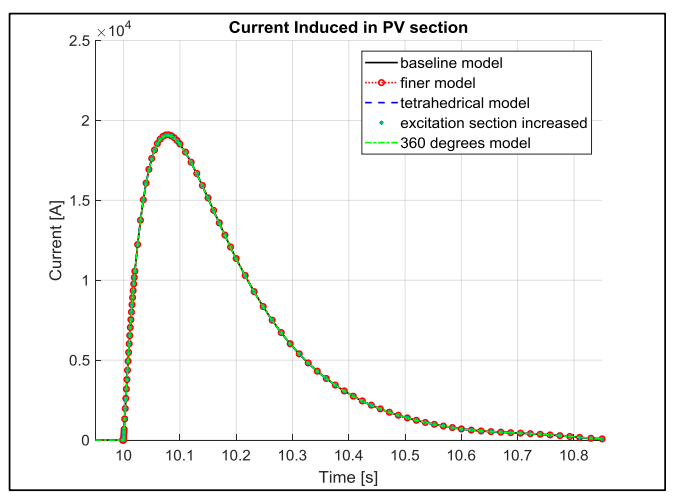

Fig. 2: Eddy current on PV comparison

Next figures show two vector plots of eddy currents in the PV for the baseline model (Fig. 3) and $360^{\circ}$ model (Fig. 4). The FE model is shown on left part of each figure. The induced eddy current in PV follows the plasma bootstrap current that mainly runs in toroidal direction, and the highest eddy current density is in the area closest to the plasma current.

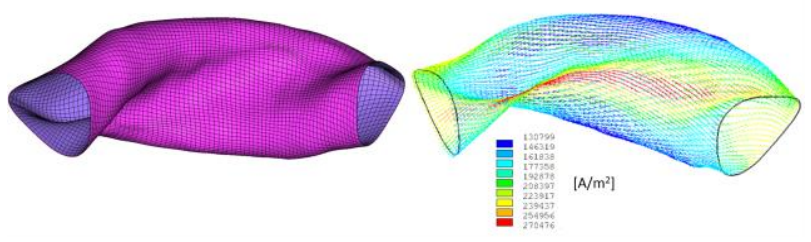

Fig. 3: Vector plot of eddy currents for baseline model

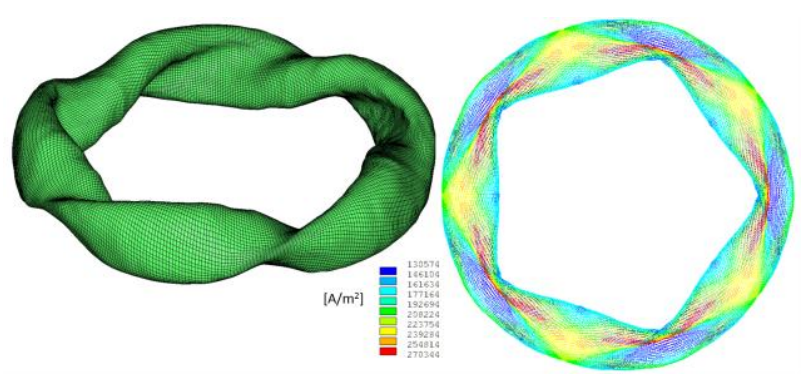

Fig. 4: Vector plot of eddy currents for $360^{\circ}$ model

\section{Preparation of the model for introduction of ports}


The activity includes an important meshing task to develop a FE model of the PV 'adapted' to the inclusion of all or selected set of ports.

Fig. 5 shows the FE model of five sectors of the PV in which the ports 'footprints' are also modelled. The same PV with all ports is shown in Fig.6. From Fig. 5 it is clearly visible that, while the mesh used in the baseline model (whether hexahedral or pentahedral) was very regular and uniform in terms of elements shape and size (Fig. 4), the mesh generated in this phase shows strong in-homogeneities.

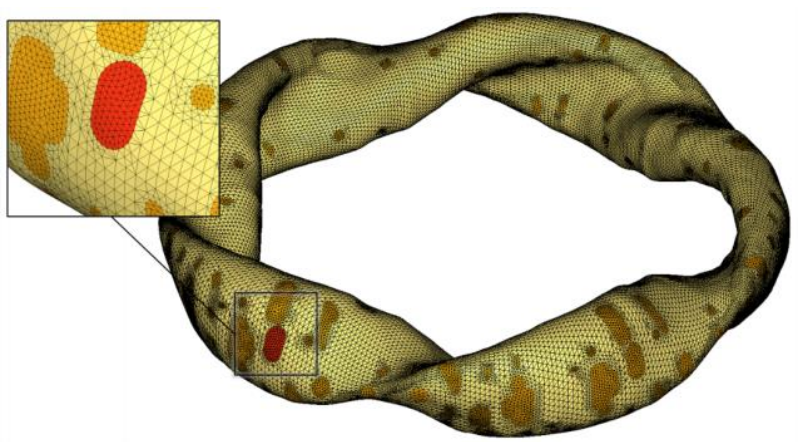

Fig. 5. FE model of five sectors PV prepared for the port inclusion

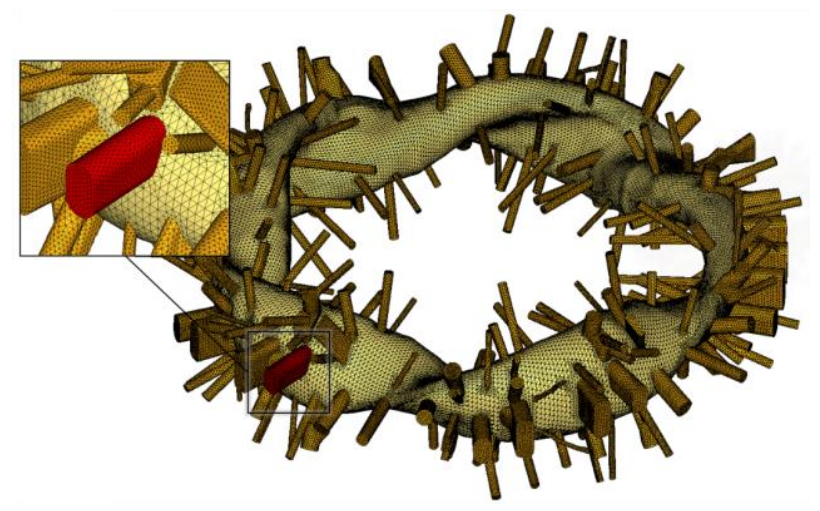

Fig. 6. FE model of five sectors PV with ports

The effect of the PV re-meshing (shows in Fig. 5 in the left box) on the eddy currents induced in the PV was evaluated on one sector models. It turned out that effects can be considered quite small (in the order of $1.5-2 \%$ ).

\section{Effect of multiple ports on PV eddy currents}

As shown in Fig. 6, all the ports are modeled from the PV to their bellows and closed end openings are introduced in the FE model. Some ports were further simplified for easy modelling, e.g. homogenize thickness were assumed with specified equivalent resistivity.

A procedure was developed in MATLAB to allow users create custom FE model by selecting the individual ports (in an Excel table) that to be included in (or excluded from) the analyses. In addition, the toroidal extension of the FE model can be 72,144 or 360 degrees. This means the possibility to select one, two or five sectors. The consideration of only 2 neighboring sectors was relevant here since a few ports are located across their boundary.

For one or two sectors models, dedicated cyclic boundary conditions were defined on the nodes belonging to the planes delimiting it in toroidal direction. It is important to remark that such periodic boundary conditions implicitly introduce in the model other "ghost ports" that equal to ports physically modelled.

In general, the above routine allows to generate an $n$ sectors model (with $\mathrm{n}=1,2,5$ ) in which each of the $254 / 5 * \mathrm{n}$ ports can be introduced or removed individually.

The intensive benchmarking activity was devoted to FE models with different number of sectors without ports. Current flowing in PV sections was evaluated for one, two and five sectors models. The maximum values of total eddy current induced in PV are reported in Table 2 and compared with maximum value obtained for 'baseline' model (Fig. 1).

Table 2. Maximum values of total eddy current in PV, without ports

\begin{tabular}{ccc}
\hline Model & Max current [A] & Delta [\%] \\
\hline${ }^{\mathrm{a}}$ One sector & 19070 & -- \\
(from phase1) & 19082 & $<0.1$ \\
One sector & 18738 & -1.74 \\
Two sectors & 19000 & -0.37 \\
$\begin{array}{l}\text { Five sectors } \\
\text { b One sector } \\
\text { (OP1.2b } \\
\text { event) }\end{array}$ & 16120 & -15.6 \\
\hline baseline model result for comparison. \\
$1.2 \mathrm{~b}$ event (initial plasma current 20 kA, time constant 2.8 ms).
\end{tabular}

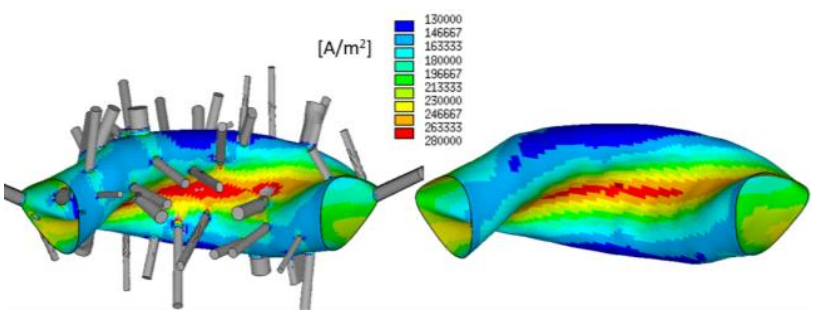

Fig. 7. Contour plot of current densities in one sector model with and without ports
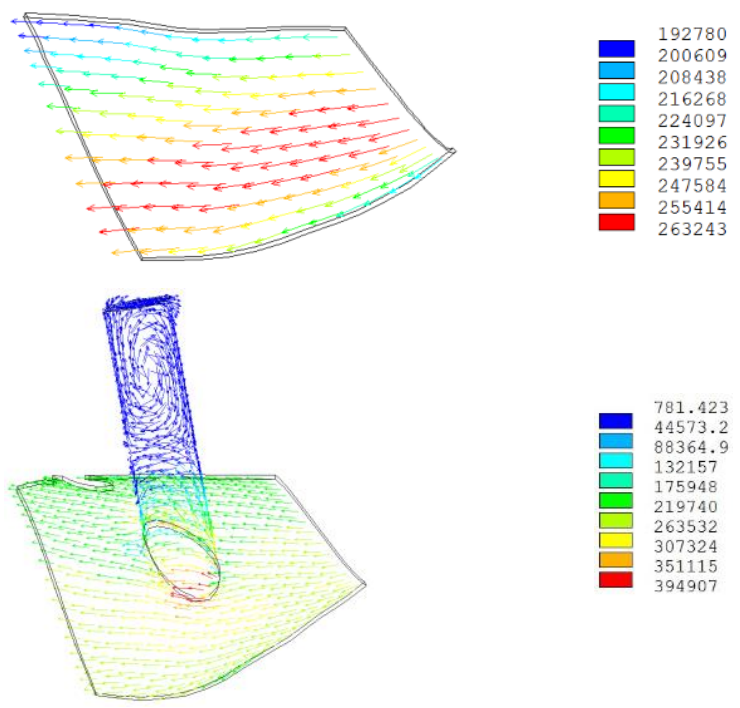
Fig. 8. Vector plot of current densities fragment in cases "no ports" (top) and with all ports (bottom) in the same location, $\mathrm{A} / \mathrm{m}^{2}$

The most impotant result of the activity is the comparison of the eddy current induced in the PV for two cases: 1) no ports and 2) all ports selected. Fig. 7 shows this comparison for one model sector. In paticular, the eddy current inside the port with maximum eddy current concentration at the neighboring PV area is of great interest. Fig. 8 indicates that the eddy current inside the port are relatively low, while the maximum current density in the PV is increased locally by about 50 $\%$ comparing with "no ports" case.

In Table 3, maximum values of total eddy current induced in the PV for one sector model with and without all ports is reported. It is reasonable that the presence of all ports reduces the total eddy current in PV due to the fact that equivalent resistance is increased by $4 \sim 7 \%$ (depending on the voltage used for the resistance calculation, since it is not constant on the section). The resistance increase is evaluated with additional electrical analysis with and without all ports of one PV module.

Table 3. Maximum values of total eddy current in PV, one sector with / without all ports

\begin{tabular}{lcc}
\hline Model & Max current [A] & Delta [\%] \\
\hline No ports & 19070 & -- \\
With all ports & 18185 & -4.62 \\
\hline
\end{tabular}

\section{Example of current sharing calculation between $P V$ and the attached components}

The analysis results indicate that the eddy currents in PV due to plasma current decay are mainly following the plasma currents direction, i.e. in toroidal direction, which provides a possibility of estimating the current sharing effect between PV and the electrically attached in-vessel components using a fragment of the PV FE model and the attachments of components to be analyzed.

As an example, the procedure has been implemented for cryo-pump electromagnetic forces calculation with the consideration of currents sharing with the PV [4]. The FE model of cryo-pump attached to a piece of the PV model were performed for electrical analysis, from which the currents divert into cryo-pump could be estimated. After that, the Lorenz forces due to the interaction with external field could be defined with a MATLAB procedure as presented in [4].

\section{Conclusions}

A set of complex EM FE models have been created to support eddy current calculation for sophisticated W7-X plasma vessel with 254 ports.

The sensitivity analyses were performed to study the influence of the PV elements size, the elements topology and the size of the $3 \mathrm{D}$ region used to model the excitation current. It turned out that the results are substantially independent from the element type, the element size and the excitation radius in selected parameter ranges (e.g. the difference in total eddy current running around the $\mathrm{PV}$ is less than $0.2 \%$ ).

The effect of the re-meshing operations in the preparation for FE modelling of 254 ports on the induced eddy currents was found to be quite small also (in the order of $1.5-2 \%$ ).

Current density in ports is generally much lower (by a factor of 2-3, at least) than the current density in the region around its attachment to the $\mathrm{PV}$.

A special routine was developed to select only required W7-X modules and ports. That allows to reduce the computational time significantly. The activity included complex adjustment of boundary conditions with dissimilar mesh and was supported by detail comparison of delivered results. E.g, a comparison of maximum eddy current values has been performed for one, two and five sectors models without ports. The differences in eddy current maximum values are less than $2 \%$.

The selection procedure was applied to one sector with all ports included. The total current flowing through a $\mathrm{PV}$ section presents a maximum value that is $4.62 \%$ lower than for the case without ports.

The PV FE model performed allows to study the behavior of diagnostic systems and other components placed inside PV shell under transient phenomena that take places during bootstrap current decay.

One typical example of the procedure to re-use the eddy current analysis results is the estimation of current sharing between PV and the electrically attached invessel components as illustrated.

The developed FE models allows also fast analysis of real plasma decay event in W7-X.

\section{Acknowledgment}

This work has been carried out within the framework of the EUROfusion Consortium and has received funding from the Euratom research and training programme 2014-2018 under grant agreement No 633053. The views and opinions expressed herein do not necessarily reflect those of the European Commission.

The authors would like to thank Matthias Köppen (at present in EPCOS company, Germany) for his contribution during initial stage of the activity, and for his helpful advices on various complex technical issues.

\section{References}

[1] R. Brakel, J. Geiger, Design values for plasma currents and time scales for current change, W7-X project document, PLM: 1-A-R0006.0.

[2] ANSYS finite element code, version 14.5

[3] D. Naujoks, Plasma decay times and ECCD operation, W7-X project document, PLM: 1-NBF-Y0065.0.

[4] J. Zhu, V. Bykov, et al., Refined multiphysics analysis of W7-X cryopumps, IEEE Transactions on plasma science 46 (2018) 1592-1602. 